\title{
Author Correction: Laboratory studies on the viability of life in $\mathrm{H}_{2}$-dominated exoplanet atmospheres
}

S. Seager (1D, J. Huang (D), J. J. Petkowski (iD and M. Pajusalu (i)

Correction to: Nature Astronomy https://doi.org/10.1038/s41550-020-1069-4, published online 4 May 2020.

In the version of this Article originally published, the sentence 'A planet that accreted from Fe-rich primitive material (for example, similar to $\mathrm{EH}$ chondritic meteorites) and water ice may have an atmosphere with up to a few per cent by mass $\mathrm{H}_{2}$, if all the iron and water reacted $^{2,3}$ ' in the first paragraph of the main text was incorrect; it should have read 'A planet that accreted from Fe-rich primitive material (for example, similar to $\mathrm{EH}$ chondritic meteorites) and water ice may have an $\mathrm{H}_{2}$-dominated atmosphere up to a few percent of the total planet mass, if all the iron and water reacted ${ }^{2,3}$. This has now been corrected.

Published online: 14 May 2020

https://doi.org/10.1038/s41550-020-1124-1

(c) Springer Nature Limited 2020 